\title{
Reduction Energy in Warped Region in Alcubierre Warp Drive
}

\author{
Gianluca Perniciano* \\ Department of Physics of the University of Cagliari,Italy \\ *g.perniciano@gmail.com
}

\begin{abstract}
A solution of general relativity is presented that describes an Alcubierre [1] propulsion system in which it is possible to travel at superluminal speed while reducing the energy, by an arbitrary value.

\section{Introduction:}

Alcubierre [1] in 1994 proposed a solution of the equations of general relativity which provides the only viable means to accelerate a spaceship up to superluminal velocities without using wormholes. A problem was soon identified: Pfenning [4] showed that the required energy is comparable to the total energy of the universe and that it is negative. We presented a way to reduce the amount of energy, involved and its spacial distribution within the warp bubble.
\end{abstract}

Note: In the following we adopt the notation used by Landau and Lifshitz in the second volume ("The Classical Theory of Fields") of their well known Course of Theoretical Physics [12].

Computazion with MAPLE 18,grTensor II. 
We propose the use of the metric

$$
d s^{2}=\left(1-v^{2} \frac{f(x, y, z-k(t))^{2}}{a(x, y, z-k(t))^{2}}\right) d t^{2}+2 v \frac{f(x, y, z-k(t))}{a(x, y, z-k(t))} d t d z-d x^{2}-d y^{2}-d z^{2}
$$

or in implicit form:

$$
d s^{2}=d t^{2}-\left[d z-v \frac{f(x, y, z-k(t))}{a(x, y, z-k(t))} d t\right]^{2}-d x^{2}-d y^{2}
$$

while Miguel Alcubierre solutions [1] is:

$$
\begin{aligned}
& d s^{2}=d t^{2}-[d z-v f(x, y, z-k(t)) d t]^{2}-d x^{2}-d y^{2} \\
& \text { and } \frac{d k(t)}{d t}=v=\text { const }
\end{aligned}
$$

Einstein Equation:

$$
G^{i k}=\frac{8 \pi G}{c^{4}} T^{i k} \quad T^{i k} \text { (energy-impulse tensor) [12] }
$$


1 Computation of the negative energy in the Pfenning zone, its comparison with the Casimir effect (plane-parallel condenser).

The energy is:

$$
E=\iiint(-g)^{1 / 2} T^{t t} d x^{3}
$$

where the triple integral extends over all the volume

and the energy density $\rho$ is:

$$
\rho=T^{t t}=k G^{t t}
$$

( $g$ determinant of the spacial metric) and $k=c^{4} / 8 \pi G$.

in Alcubierre form:

$$
\rho=T^{t t}=k G^{t t}=-k \frac{1}{4} v^{2} \frac{x^{2}+y^{2}}{r^{2}}\left(\frac{\partial h}{\partial r}\right)^{2}
$$

$$
\text { where } h=h(r)=\frac{f(r)}{a(r)}
$$

and $\quad k=c^{4} / 8 \pi G ; c$ is the speed of light and $\mathrm{G}$ is Newton's gravitational constant;

$$
G^{t t}=-\frac{1}{4} v^{2} \frac{x^{2}+y^{2}}{r^{2}} g(r)
$$

where $g(r)$ is given by:

$$
g(r)=\left[\frac{1}{a(r)^{2}}\left(\frac{d f(r)}{d r}\right)^{2}+\left(\frac{f(r)^{2}}{a(r)^{4}}\right)\left(\frac{d a(r)}{d r}\right)^{2}-2 \frac{d f(r)}{d r} \frac{f(r)}{a(r)^{3}} \frac{d a(r)}{d r}\right]
$$




\subsection{The energy $E$ in the Pfenning (warped region) zone in our case (see [4] for an example):}

The energy given by :

$$
E=-\frac{8 \pi k v^{2}}{32 \pi} \int \frac{x^{2}+y^{2}}{r^{2}} g(r) d x^{3}
$$

- 1)-inside the warp bubble $f(r)=1$ and $a(r)=1$

- 2)-outside the warp bubble $f(r)=0$ and $a(r)=1$

- 3)-within the Alcubierre warped region $0<f(r)<1$ and $a(r) \gg 1$ they are (12),(13)

$$
f(r) \text { is for all } r \text { (example): }
$$$$
f(r)=(1 / 2)(1-\tanh @(r-R)),(d f / d r)^{2}=(1 / 4)\left(@^{2} /\left[(\cosh @(r-R))^{4}\right]\right)
$$

$$
a(r) \text { is for all } r \text { (example): }
$$

$$
a(r)=\left(2^{P}\right) /\left[1+(\tanh [@(r-R)])^{2}\right]^{P} \quad P \gg 1
$$

$$
\begin{aligned}
& d a(r) / d r=(-P)\left((1 / 2)\left[1+(\tanh [@(r-R)])^{2}\right]\right)^{-(P+1)}\left(@ \tanh [@(r-R)] /(\cosh [@(r-R)])^{2}\right) \\
& (d a(r) / d r)^{2}=(-P)^{2}\left((1 / 2)\left[1+(\tanh [@(r-R)])^{2}\right]\right)^{(-2(P+1))}\left(@ \tanh [@(r-R)] /(\cosh [@(r-R)])^{2}\right)^{2}
\end{aligned}
$$

$R \quad$ radius of the warp bubble

$$
\begin{gathered}
\text { Equation (10): } g(r)=\left[\frac{1}{a(r)^{2}}\left(\frac{d f(r)}{d r}\right)^{2}+\left(\frac{f(r)^{2}}{a(r)^{4}}\right)\left(\frac{d a(r)}{d r}\right)^{2}-2 \frac{d f(r)}{d r} \frac{f(r)}{a(r)^{3}} \frac{d a(r)}{d r}\right] \\
g(r)=g_{1}(r)+g_{2}(r)+g_{3}(r) \\
g_{1}(r)=\frac{1}{a(r)^{2}}\left(\frac{d f(r)}{d r}\right)^{2}=\left(\frac{1}{4}\right) \frac{@^{2}}{a(r)^{2}(\cosh [@(r-R)])^{4}}
\end{gathered}
$$




$$
\begin{gathered}
g_{2}(r)=\left(\frac{f(r)^{2}}{a(r)^{4}}\right)\left(\frac{d a(r)}{d r}\right)^{2} \approx(P @)^{2} \frac{(\tanh [@(r-R)])^{2}}{a(r)^{2}(\cosh [@(r-R)])^{4}} \\
g_{3}(r)=-2 \frac{d f(r)}{d r} \frac{f(r)}{a(r)^{3}} \frac{d a(r)}{d r} \approx-\left(P @{ }^{2}\right) \frac{\tanh [@(r-R)]}{a(r)^{2}(\cosh [@(r-R)])^{4}}
\end{gathered}
$$

for $\mathrm{P}=280, @=5000, a(r) \gg 1$ in Alcubierre warped region, the energy density is,for equations (15),(16),(17), and paper [11],[13],[14]:

$$
\text { energy density }=\left|-\frac{k}{4} v^{2} \frac{x^{2}+y^{2}}{r^{2}} g(r)\right| \ll 1
$$

in Alcubierre warped region.

And if $R=100 \mathrm{~m} \quad P=280, \quad @=5000$ [14],in Alcubierre warped region (there is the source of esotic matter), the energy is,for equation (5):

$$
E=-\frac{8 \pi k v^{2}}{12} \int_{0}^{+\infty} r^{2} g(r) d r \quad k=c^{4} / 8 \pi G
$$

and for the same values of the parameters shown above the Energy is:

$$
E \approx-4 v^{2} 10^{-87} \text { joule }
$$

energy is very small compared with Casimir effect (23).

And mass is :

$$
m \approx-4 v^{2} 10^{-104} \mathrm{~kg}
$$

If $R=100 \mathrm{~m} \quad P=140, @=5000 \quad$ [14],in Alcubierre warped region (there is the source of esotic matter), the energy is:

$$
E \approx-v^{2} 10^{-7} \text { joule }
$$

energy is compared with Casimir effect, with $\mathrm{d}=100 \mathrm{~nm}$ and $\mathrm{L}=1 \mathrm{~m}$, equation (23). 
For possibly,esotic matter see also,example [16].

A modification of parameters $\mathrm{P}, @$, in a(r) and for $\mathrm{f}(\mathrm{r})$, between those chosen, determines a greater or lesser reduction of the energy density and the energy,with the same radius value $\mathrm{R}$ of the warp bubble that contains the spaceship, and also changes the thickness of the warped region.

\subsection{The energy $U(d)$ between the two plates in a plane-parallel condenser in empty space, due} to the Casimir effect, is:

$$
U(d)=-\pi^{2}\left[\left(\frac{h}{2 \pi}\right) \frac{c}{720 \mathrm{~d}^{3}}\right] L^{2}
$$

where $\mathrm{d}$ is the distance between the plates and $\mathrm{L}$ is the side of the square conducting plate. As can be seen, the energy is negative and this implies that the force (equal to the opposite of the derivative with respect to $d$ ) is attractive, as has been experimentally found ("Lamoreaux" [15]). In the case $d=1 \mu \mathrm{m} \quad$ and $\mathrm{L}=1 \mathrm{~m}$ ( $\mathrm{L}$ is chosen to be quite large, but not as large as the Pfenning zone) it is found that $U(d)=-410^{-10}$ joule .

\section{Conclusions}

These calculations show that the modified Alcubierre propulsion system can achieve superluminal speeds, and the energy,can be reduced by an arbitrary value in the Pfenning zone, warped region, (source of esotic matter) 


\section{References}

[1] M. Alcubierre, Classical and Quantum Gravity 11, L73 (1994).

[2] C. Barcelo, S. Finazzi, and S. Liberati, ArXiv e-prints (2010), arXiv:1001.4960 [gr-qc].

[3] C. Clark, W. A. Hiscock, and S. L. Larson, Classical and Quantum Gravity 16, 3965 (1999).

[4] M. J. Pfenning and L. H. Ford, Classical and Quantum Gravity 14, 1743 (1997),arXiv:9702026

[5] F. S. N. Lobo and M. Visser, Classical and Quantum Gravity 21, 5871 (2004).

[6] F. S. N. Lobo, ArXiv e-prints (2007), arXiv:0710.4474 [gr-qc].

[7] Finazzi, Stefano; Liberati, Stefano; Barceló, Carlos (2009). "Semiclassical instability of dynamical warp drives".Physical Review D 79 (12): 124017. arXiv:0904.0141

[8] Van den Broeck, Chris (1999). "On the (im)possibility of warp bubbles". arXiv:gr-qc/9906050

[9] C. Van Den Broeck, Class. Quantum Grav. 16 (1999) 3973

[10] Hiscock, William A. (1997). "Quantum effects in the Alcubierre warp drive spacetime".

Classical and Quantum Gravity 14 (11): L183-L188. arXiv gr-qc/9707024

[11] Perniciano G.(2015),viXra:1507.0193

[12] L D Landau and E M Lifshitz “The Classical Theory of Fields”, Fourth Edition: Volume 2 (Course of Theoretical Physics Series)

[13] Perniciano G. (2015),viXra:1507.0165

[14] Loup F. (2015),hal-01183043,viXra:1508.0048

[15]S. K. Lamoreaux, "Demonstration of the Casimir Force in the 0.6 to $6 \mu$ m Range",Phys. Rev. Lett.78,5-8 (1997)

[16] Negative mass hydrodynamics in a spin-orbit-coupled Bose-Einstein condensate, arXiv condmat-quantum-gas/1612.04055,(2016) 